\title{
Removal of a red tattoo on the lips using a 532-nm picosecond laser
}

Syeo Young Wee ${ }^{1}$, Tae Hyung Kim ${ }^{1}$, Eun Soo Park ${ }^{2}$

\author{
${ }^{1}$ Department of Plastic and \\ Reconstructive Surgery, Soonchunhyang \\ University Gumi Hospital, Gumi; \\ ${ }^{2}$ Department of Plastic and \\ Reconstructive Surgery, Soonchunhyang \\ University Bucheon Hospital, Bucheon, \\ Korea
}

This work was supported in part by the Soonchunhyang University Research Fund.
Tattoos, which people choose to have performed for various reasons, can have multiple colors, ranging from conventional black to red, yellow, blue, and others. As tattoos have become increasingly popular, the need for tattoo removal has also grown, and the most commonly used method for removal is a laser. However, the extent to which various types of lasers remove different tattoo pigments is clearly important. Although extensive research has been done on black tattoos, red tattoos have not been adequately studied, and there are few case reports on red tattoos. We present a case of effective removal of a red tattoo from the lips using a picosecond laser.

Keywords Laser / Tattooing / Pigmentation

\section{INTRODUCTION}

Tattooing of the face for cosmetic purposes is generally done to conceal a scar on the face, to make one's lips look fuller, to create a line on the eyes or lips, and in recent years, to establish a permanent shape of the eyebrows [1]. However, despite the popularization of tattooing, people often seek tattoo removal if the tattoo is not to their liking, is not in line with current fashion, or for other reasons. Efforts to remove tattoos have always produced variable results. However, debate continues regarding whether laser removal is the most effective method, and it has yet to be established to what extent different types of lasers remove certain types of tattoos. Many studies have reported the removal of black tattoos, but there are insufficient data on the efficacy of laser removal of red tattoos. Herein, we report a case in which we obtained good results using a picosecond laser for a red tattoo on the lips.

Received: Mar 2, 2021 Revised: Mar 31, 2021 Accepted: Apr 2, 2021 Correspondence: Eun Soo Park Department of Plastic and Reconstructive Surgery, Soonchunhyang Bucheon Hospital, 170 Jomaru-ro, Wonmi-gu, Bucheon 14584, Korea

Tel: +82-32-621-5319, Fax: +82-32-621-5016, E-mail: peunsoo@schmc.ac.kr Copyright () 2021 The Korean Society for Aesthetic Plastic Surgery.

This is an Open Access article distributed under the terms of the Creative Commons Attribution Non-Commercial License (https://creativecommons.org/licenses/by-nc/4.0/) which permits unrestricted non-commercial use, distribution, and reproduction in any medium, provided the original work is properly cited. $\quad w w w . e-a a p s . o r g$

\section{CASE REPORT}

A 53-year-old female patient visited our clinic for the removal of an abnormally placed and asymmetric red-pigmented tattoo on her lips; she had Fitzpatrick skin type 1. The tattoo on the lips was pigmented with a relatively weak red color from the lips to the outer line, including the dark outer line (Fig. 1). The patient had not undergone any treatments for tattoo removal since she received the tattoo 5 years previously. She had no other medical history.

Before the procedure, $5 \%$ lidocaine topical anesthetic ointment (Emla; AstraZeneca AB, Karlskoga, Sweden) was applied at the treatment site for 30 minutes. It was washed off using soap and water before the procedure. A PICO laser (Picocare; Wontech Co., Daejeon, Korea) with a wavelength of $532 \mathrm{~nm}$ was used.

The patient underwent laser treatment 5 times at an average interval of 3.5 weeks over the course of 1.5 years, from March 2017 to July 2019. Each session was performed with a fluence of $0.8-1.0 \mathrm{~J} / \mathrm{cm}^{2}$ and a repetition rate of $2-10 \mathrm{~Hz}$ using a 3-mm spot size (Table 1). For the dark outline and the inner lighter color, different repetition rates were set for the second procedure, and each time, the fluence, repetition rate, and the number of shots were determined based on the color status.

After each session, an ice pack was applied immediately, and a topical broad-spectrum antibiotic ointment was prescribed. The patient was instructed to avoid sun exposure and to use sunblock.

After the procedure, temporary mild erythema and edema were observed; however, there was no scar formation or other severe ad- 
Table 1. Protocol of laser treatment

\begin{tabular}{|c|c|c|c|c|c|}
\hline Procedure & Interval between procedures & Spot size $(\mathrm{mm})$ & Fluence (J) & Repetition (Hz) & Shot count (shot) \\
\hline Second & 9 Months & 3 & 1.0 & 9 (outer line) & 612 (outer line) \\
\hline Third & 1 Year, 6 months & 3 & 0.8 & 10 & 1,228 \\
\hline Fourth & 7 Weeks & 3 & 1.0 & 10 & 441 \\
\hline
\end{tabular}

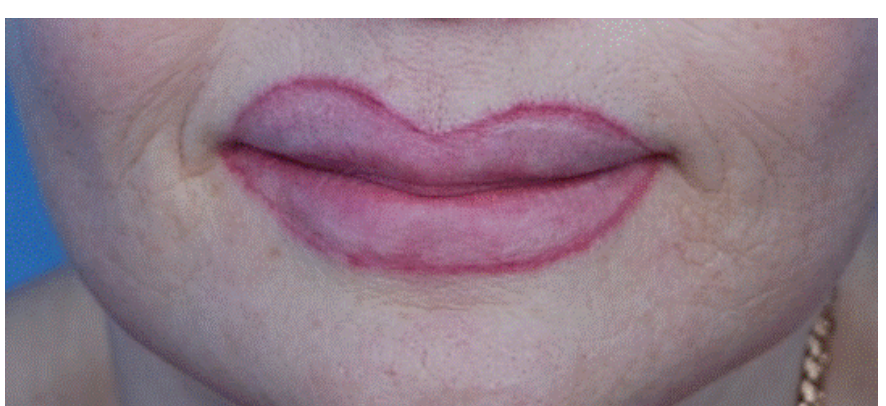

Fig. 1. Photographic findings before the first laser treatment.

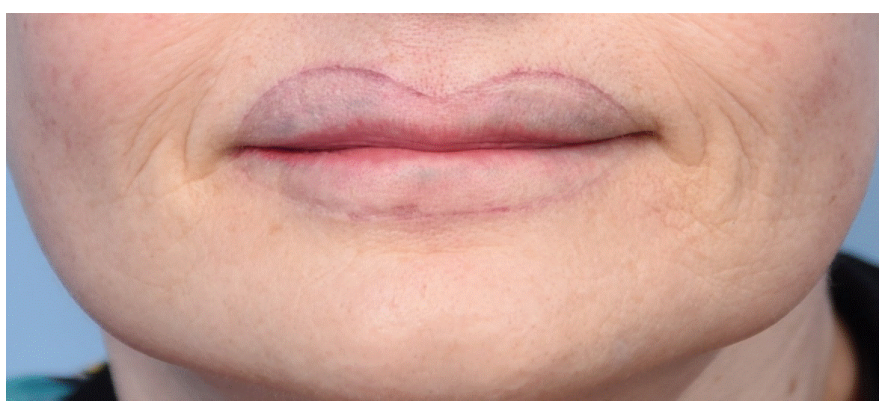

Fig. 2. Photographic findings after two sessions of laser treatment.

verse effects such as paradoxical darkening (Fig. 2).

Photographs taken before each session were evaluated by the investigator to measure the percentage of clearance. No clearance was specified as $0 \%$, and complete removal of the tattoo was specified as $100 \%$.

After two sessions, 70\% clearance of the tattoo was achieved (Fig. 2). After the final five sessions over 2 years, there was more than $90 \%$ clearance (Fig. 3). No adverse effects were observed during an additional follow-up period of 1 year.

\section{DISCUSSION}

Previously, procedures such as dermabrasion, cryosurgery, and classical excision were performed for the removal of tattoos. More recently, carbon dioxide lasers have been introduced; however, this technique can leave a scar [1]. Selective photothermolysis with la-

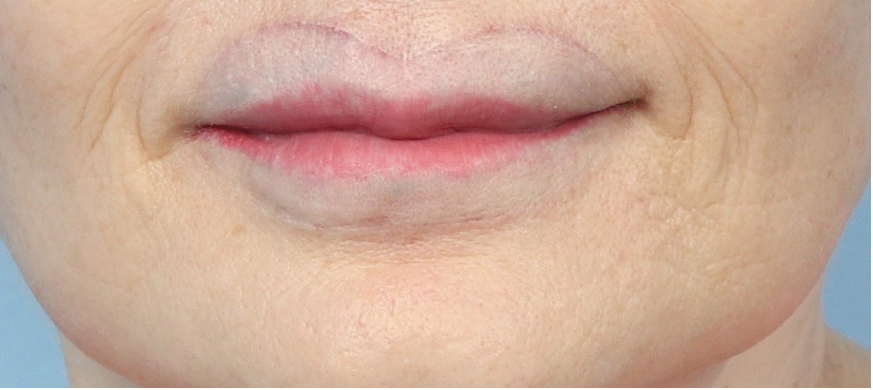

Fig. 3. Photographic findings after five sessions of laser treatment.

sers, as a newer technique, effectively removes the tattoo pigment [2]. The tattoo pigment functions as a target chromophore that responds to a particular wavelength [1]. For the target chromophore to be adequately obliterated, the pulse duration must be shorter than the thermal release time; however, the thermal release time of the tattoo pigment is less than 10 nanoseconds, and since the tattoo particles are small, a short pulse duration is required $[3,4]$.

The Q-switched laser was used for tattoo removal by delivering high-powered, short-duration pulses [3]. However, the diameter of tattoo particles $(20-200 \mathrm{~nm})$ is smaller than that of melanosomes, necessitating the use of picosecond lasers [5]. A picosecond laser generates a substantial photomechanical effect and a high peak temperature using a short pulse duration, providing an effective treatment with low fluence [6]. The shorter pulses delivered by picosecond lasers are known to remove tattoo particles more effectively than the longer pulses delivered by nanosecond lasers $[7,8]$.

Various colors of tattoos, including black, dark blue, green, and brown, are known to respond well to lasers; however, red, orange, yellow, and light blue are difficult to remove [3]. The laser wavelengths that react with pigments of each color are known: a 694$\mathrm{nm}$ laser reacts with green pigments (and, to a lesser extent, black and blue pigments), a 755-nm laser with blue pigments (black, green), and 1064-nm laser with black (blue) pigments, while a 532$\mathrm{nm}$ laser reacts with red, orange, purple, brown and yellow tattoo pigments $[3,5,9,10]$.

According to previous studies on red tattoos, a 532-nm picosecond laser was more effective for removal than a 532/1064-nm nanosecond laser and a 1064-nm picosecond laser [5]. However, in some 
cases, laser treatment on red tattoos can cause paradoxical darkening, wherein ferric oxide in the tattoo pigment turns into ferrous oxide by oxidation, which causes the color to become darker [3]. Therefore, laser treatment for red tattoos needs careful consideration.

The patient in our case achieved nearly complete removal of a red tattoo through treatment with a 532-nm picosecond laser. However, due to the personal circumstances of the patient, she was unable to undergo the procedure at fixed intervals, which might be a limitation of using this case to create a generalized protocol for the procedure. Moreover, as our patient had Fitzpatrick skin type 1, it is not possible to predict outcomes of this procedure for other skin types.

Nevertheless, this case report demonstrates useful guidelines for the removal of red tattoos, which are difficult to remove with conventional treatment. In particular, the use of a picosecond laser should be considered to remove red tattoos, which are difficult to remove through laser treatment.

\section{NOTES}

\section{Conflict of interest}

Eun Soo Park is an editorial board member of the journal but did not involve in the peer reviewer selection, evaluation, or decision process of this article. No other potential conflicts of interest relevant to this article were reported.

\section{Ethical approval}

The study was retrospectively approved by the Institutional Review Board of Soonchunhyang University Bucheon Hospital (IRB No. 2021-02-003-001) and performed in accordance with the principles of the Declaration of Helsinki.

\section{Patient consent}

The patient provided written consent for the use of her images.

\section{ORCID}

Syeo Young Wee

https://orcid.org/0000-0002-1787-9715

Tae Hyung Kim

https://orcid.org/0000-0001-9537-2330

Eun Soo Park

https://orcid.org/0000-0003-2966-9122

\section{REFERENCES}

1. Kuperman-Beade M, Levine VJ, Ashinoff R. Laser removal of tattoos. Am J Clin Dermatol 2001;2:21-5.

2. Anderson RR, Parrish JA. Selective photothermolysis: precise microsurgery by selective absorption of pulsed radiation. Science 1983;220: 524-7.

3. Henley JK, Zurfley F, Ramsey ML. Laser tattoo removal. In: StatPearls [Internet]. Treasure: StatPearls Publishing; 2020.

4. Spicer MS, Goldberg DJ. Lasers in dermatology. J Am Acad Dermatol 1996;34:1-25.

5. Kono T, Chan HHL, Groff WF, et al. Prospective comparison study of $532 / 1064 \mathrm{~nm}$ picosecond laser vs 532/1064 $\mathrm{nm}$ nanosecond laser in the treatment of professional tattoos in Asians. Laser Ther 2020;29:4752.

6. Saedi N, Metelitsa A, Petrell K, et al. Treatment of tattoos with a picosecond alexandrite laser: a prospective trial. Arch Dermatol 2012;148: 1360-3.

7. Bernstein EF. Laser tattoo removal. Semin Plast Surg 2007;21:175-92.

8. Ross V, Naseef G, Lin G, et al. Comparison of responses of tattoos to picosecond and nanosecond Q-switched neodymium: YAG lasers. Arch Dermatol 1998;134:167-71.

9. Stafford TJ, Lizek R, Boll J, et al. Removal of colored tattoos with the Q-switched alexandrite laser. Plast Reconstr Surg 1995;95:313-20.

10. Alabdulrazzaq H, Brauer JA, Bae YS, et al. Clearance of yellow tattoo ink with a novel 532-nm picosecond laser. Lasers Surg Med 2015;47: 285-8. 